\title{
SUMMARY OF PRESENTATIONS: FIRST SESSION
}

\section{EDITOR'S NOTE}

The papers summarized here discuss emerging infectious diseases in terms of two categories: associated modes of transmission and antibiotic resistance. In the former category, four presentations examine disease manifestation, epidemiologic trends, and factors surrounding emergence and identification of many recently emerged infectious diseases. The first presentation discusses Cryptosporidium as a paradigm for waterborne emerging pathogens. The second includes data on foodborne diseases, discusses established foodborne disease agents and emerging foodborne pathogens, and concludes with factors that contribute to the emergence of foodborne pathogens.

The third paper summarized here surveys two airborne pathogens: Legionellae and the hantaviruses; it concludes with guidelines for a response to, and surveillance of, airborne infectious diseases. The final paper reproduced for this category deals with sexually transmitted diseases. It provides an overview of current epidemiologic data on such diseases and also examines trends in the emergence of antibiotic resistance among these organisms.

The topic of antibiotic resistance was examined in several presentations. Three are summarized here.

As fewer antibiotics become available for treatment, antibacterial and antiviral strategies are needed to minimize their need and to ensure their proper use. Such strategies should emphasize (1) infection control; (2) general public health promotion; (3) immunization; (4) new treatments, which should include treating underlying diseases involved in host defenses; and (5) the education of hospitals, the public, and medical professionals regarding proper treatment with antibiotics.

Pharmaceutical companies are targeting particular areas and organisms to provide rapid treatment. Gene therapy and potentiators are being pursued, although products employing such technology will not emerge in the near future. Approximately 100,000 compounds are screened every year and matched with organisms. A large number of 
these compounds are botanical derivatives. Three nosocomial pneumonia compounds are currently awaiting Food and Drug Administration approval.

The papers summarized in this issue include presentations on methicillin-resistant Staphylococcus aureus, penicillin-resistant pneumococci, and hospital-and community-acquired gram-negative pathogens. The final paper in the summary of the first day presents data on the increasing incidence of vancomycin-resistant enterococci in New York City, New York State, and the US. It also examines several questions that should be addressed regarding vancomycin-resistant enterococci.

\section{CATEGORY: ASSOCIATED MODES OF TRANSMISSION}

TOPIC: WATERBORNE PATHOGENS

PRESENTER: ROSEMARY SOAVE, MD, ASSOCIATE PROFESSOR

OF MEDICINE AND PUBLIC HEALTH, CORNELL UNIVERSITY MEDICAL COLLEGE*

As demonstrated by an April 1993 outbreak in Milwaukee, Wisconsin, which infected over 400,000 people and killed more than 100 mostly immunocompromised victims, Cryptosporidium is a waterborne pathogen with the potential to cause massive municipal outbreaks. It has a chlorine contact time 640 times that of Giardia lamblia, which makes the oocyst of Cryptosporidium resistant to chlorination by municipal water supplies and gives it the ability to survive in the natural environment with little nutrition for many months. After ingestion by a human host, the oocyst undergoes exocystation in the intestine, releasing a motile, much more fragile sporozoite. After developing into protozoites and then forming merozoites, the organism enters its sexual phase; this phase carries with it the capacity for autoinflection.

This "recycling," or continual reinfection by the merozoites, reaches its epitome in immunocompromised patients-such as patients with human immunodeficiency virus (HIV) infection, people with immunoglobulin A deficiency or chronic hypogammaglobunemia, and those chronically immunosuppressed by steroids following cancer therapy.

Due to sustained cholera-like diarrhea, immunosuppressed individuals shed enormous numbers of organisms into the environment: from 1.2 million to 12 billion organisms per day. It is not known how the pathogen causes diarrhea, but the watery nature and the lack of inflammatory changes in the host both suggest toxin mediation.

Many species of animals can be infected with Cryptosporidium. There are

${ }^{*}$ Dr. Soave's address is Division of Infectious Diseases, New York Hospital-Cornell Medical Center, 1300 York Avenue, Box 125, New York, NY 10021. 